\title{
RESEARCH
}

\section{Minimal sufficiency with covert even}

\author{
Daniele Panizza ${ }^{1}$ and Yasutada Sudo² \\ ${ }^{1}$ Georg-August-Universität Göttingen, Göttingen, DE \\ 2 University College London, London, GB \\ Corresponding author: Yasutada Sudo (y.sudo@ucl.ac.uk)
}

Exclusive particles (e.g. just) express exclusivity inferences by negating focus alternatives to the sentence they modify. Grosz (2012) observes that they can sometimes give rise to what he calls minimal sufficiency readings, which seem to affirm, rather than negate, focus alternatives. Grosz proposes to analyze them in terms of the rank-order reading, a kind of scalar reading of exclusive particles that is independently attested. Coppock \& Beaver (2014) put forward a similar analysis based on their unified semantics for different uses of exclusive particles. We point out that these previous accounts fail to capture the distribution of minimal sufficiency readings, in particular, the relevance of distributivity, and propose an alternative analysis where the scalar component of the minimal sufficiency reading comes from a covert version of even, rather than from the exclusive particle itself. Empirical support for this comes from the generalization that an overt even can be added to sentences that have minimal sufficiency readings without changing the meaning, but not to sentences that do not allow for minimal sufficiency readings. We argue that our account not only captures the distribution of the minimal sufficiency reading, but also derives the inferences involved in the minimal sufficiency reading compositionally together with the standardly assumed semantics for exclusive particles and even.

Keywords: minimal sufficiency; just; even; focus sensitivity; distributivity

\section{Introduction}

Exclusive particles like just and only are so-called because they express exclusivity inferences based on focus alternatives (Rooth 1985; 1992, among others). To illustrate, consider (1). What is in focus is marked by a subscript $\mathrm{F}$ throughout this paper.

a. Just one , $_{F}$ cat is in the room.

$\Rightarrow \neg$ (two cats are in the room), $\neg$ (three cats are in the room),...

b. Only Patrick ${ }_{F}$ is in the room.

$\Rightarrow \neg$ (Andy is in the room), $\neg($ Bojan is in the room),...

The focus alternatives of (1a) are sentences of the form ' $\xi$ cats are in the room' for a numeral in place of $\xi$, and those of (1b) are sentences of the form ' $\xi$ is in the room' where a name occupies the place of $\xi$. The rule of thumb is that the focus alternatives are derived by replacing the focused material with related, contextually relevant expressions (see Fox \& Katzir 2011 for more discussion on this). The semantic function of an exclusive particle, then, is to negate those alternatives that are not entailed by the sentence they modify (the latter of which is often called the prejacent). ${ }^{1}$ Consequently, (1a) entails that no more than one cat is in the room, and (1b) entails that no one other than Patrick is in the room.

\footnotetext{
${ }^{1}$ For ease of exposition we assume throughout this paper that focus alternatives are linguistic expressions, as proposed by Fox \& Katzir (2011), rather than semantic objects, as assumed by Rooth (1985; 1992), but as
} 
Grosz (2012) observes that exclusive particles can sometimes be used to mean essentially the opposite of an exclusivity inference, i.e. to affirm at least some alternatives not entailed by the prejacent. Following Grosz, we call such readings of exclusivity particles minimal sufficiency readings. For example, consider the following example.

Just one ${ }_{F}$ cat will make Patrick happy.

The focus alternatives of this sentence are sentences of the form ' $\xi$ cats will make Patrick happy', where $\xi$ is a numeral. Besides a reading that is similar to (1), which entails that two or more cats will not make Patrick happy, it has another, perhaps more natural, reading, from which one can infer that at least some, if not all, of the alternatives are also true, i.e. multiple cats will also make Patrick happy.

Minimal sufficiency readings are observed with various kinds of foci, and in different grammatical positions, as demonstrated by (3).

a. Just a letter ${ }_{F}$ from her makes me happy.

b. Katie gets drunk with just one $e_{F}$ pint of beer.

c. I need more than $£ 20$ just for [the train to the airport] ${ }_{F}$.

In all these examples of minimal sufficiency readings, the relevant inference is intuitively scalar and additive: Roughly, the prejacent describes a relatively unlikely/surprising case for some event to happen or some state to hold, and the sentence also suggests that some other focus alternatives that are more likely/less surprising are also true. Concretely, take the minimal sufficiency reading of (2), for example. The prejacent expresses that there being one cat leads to Patrick's happiness. The scalar part of the meaning says that it is relatively unlikely/surprising that Patrick becomes happy with exactly one cat, and the additive part of the meaning implies that there being more cats will also lead to Patrick's happiness. In the bulk of this paper we will focus mostly on (2) and (3a) as our representative examples, but our proposal can explain (3b) and (3c).

There are two previous analyses of the minimal sufficiency reading, namely, Grosz (2012) and Coppock \& Beaver (2014). As explained in detail below, according to these authors, the minimal sufficiency reading is triggered by just. We will argue, however, that while these accounts derive minimal sufficiency readings for examples like (2) and (3), they fail to capture the fact that certain examples, e.g. (1a), do not have minimal sufficiency readings.

Our main goal in this paper is to put forward an alternative account so as to better explain the distribution of minimal sufficiency readings. Our account will be based on the following distributional generalization: a minimal sufficiency reading is available exactly when an overt even can be felicitously added to mean the same thing. By way of illustration, observe that (4a) is felicitous and has the same reading as (2), while (4b) is infelicitous and (1a) lacks a minimal sufficiency reading.

a. Even just one e $_{F}$ cat will make Patrick happy.

b. \#Even just one ${ }_{F}$ cat is in the room.

We will explain in detail how the two focus sensitive particles, even and just, conspire to give rise to a reading equivalent to the minimal sufficiency reading in sentences like

far as we can see, nothing crucial in our analysis hinges on this. For the sake of uniformity, we will rephrase Grosz's (2012) and Coppock \& Beaver's (2014) analyses in Section 2 in a way that conforms to this, but none of our criticisms against these analyses relies on it. 
(4a), as well as how they render sentences like (4b) infelicitous. We will emphasize, in particular, the role of distributivity in the present phenomenon. Then, based on this, we will explain away the minimal sufficiency readings of examples like (2) and (3), as well as the lack of minimal sufficiency readings in sentences like (1a), by resorting to a covert version of even. That is, we will claim that the minimal sufficiency reading of an exclusivity particle requires covert even, and its distribution tracks the felicity of overt even. To put it differently, we reduce the problem of explaining the compositional semantics and distribution of the minimal sufficiency reading to the problem of explaining the semantics of sentences like (4) with overt even.

The organization of this paper is as follows. In Section 2 we will review the two previous analyses of the minimal sufficiency reading due to Grosz (2012) and Coppock \& Beaver (2014), and argue that they fail to correctly capture the distribution of the minimal sufficiency reading. In Section 3 we will explain how the standardly assumed semantics of just and even can be used to understand the observed readings of sentences like (4a), and claim that the minimal sufficiency reading of sentences like (2) can be explained in exactly the same way by postulating covert even. Then in Section 4, we will consider how the infelicity of sentences like (4b) can be explained, and claim that our analysis of it will also explain the lack of minimal sufficiency readings for sentences like (1a). We will discuss some additional observations about the meaning of just in Section 5, and we will conclude in Section 6.

\section{Previous analyses}

As far as we know, there are only two compositional analyses of the minimal sufficiency reading of exclusive particles, namely, those by Grosz (2012) and Coppock \& Beaver (2014). Both analyses are based on the idea that the minimal sufficiency reading is a special case of the scalar reading of exclusive particles, a reading that is independently observed for exclusive particles like ust, as in (5). ${ }^{2}$

\section{Patrick is just a postdoc ${ }_{F}$}

In this sentence just introduces a scalar inference that being a postdoc is low on some scale that is assumed to be relevant in the current context of utterance, e.g. a scale based on academic ranks. To explain this, Grosz (2012) postulates a separate lexical entry for the scalar reading of just (and other exclusivity particles across languages), which introduces a presupposition that the prejacent is low on some contextually salient scale formed by its focus alternatives (and is vacuous with respect to the at-issue content). Coppock \& Beaver (2014)aim at capturing this reading of just without postulating a separate lexical entry from the one for the exclusivity reading. In what follows we will review how they use this scalar reading of just to derive the minimal sufficiency reading, and point out their overgeneration problems.

\subsection{Grosz (2012)}

Grosz (2012: §6.2) postulates two versions of an exclusive particle, which he calls ONLY and $\mathrm{ONLY}_{2}$, and uses the latter to account for the minimal sufficiency reading (ONLY is used for the exclusive reading, and doesn't concern us here). He gives the following denotation to the $\mathrm{ONLY}_{2}$ reading of an exclusive particle (adapted from Grosz 2012: 216).

\footnotetext{
${ }^{2}$ To be more precise, Coppock \& Beaver (2014) postulate a general meaning for all uses of exclusive particles, and the exclusivity reading can be seen as a special case where the relevant scale is that of entailment (see below for details). What we mean here is that their analysis of the minimal sufficiency reading appeals to a non-entailment based scale, as it would for (5).
} 
'ONLY $S_{2}$ ' presupposes with respect to some contextually salient scale that most of the relevant focus alternatives of ' $S$ ' are higher ranked than ' $S$ ' on that scale, and asserts the same thing as ' $S$ '.

Grosz's main interest is to account for uses of exclusive particles like only in optative constructions across languages using (6), about which we have nothing to say in this article. Yet, he explicitly claims in \$6.2.2.2 that the minimal sufficiency reading of just is to be accounted for by (6) as well (while remarking at the same time that such readings of only are restricted in English; see the discussion at the end of this paper). For example, he would analyze (2), repeated here, as follows.

Just one $_{F}$ cat will make Patrick happy.

Grosz would assume that just here takes a propositional scope, and operates on focus alternatives that include (7), of which (7a) is the prejacent (Note that by convention the prejacent is included as a focus alternative to itself).
a. One cat will make Patrick happy.
b. Two cats will make Patrick happy.
c. Three cats will make Patrick happy.

Just yields a scalar presupposition with respect to some salient scale, which is presumably a scale of likelihood or expectedness in this case. Thus, the scalar presupposition amounts to that the prejacent (7a) is relatively unlikely or surprising among the focus alternatives, which captures the scalar component of the minimal sufficiency reading of this example. Note also that assuming that the numerals in these focus alternatives receive lower-bounded ('at least') readings (see Section 3.1 for more on this), (7a) entails the other alternatives, so the additive component of the minimal sufficiency reading will be captured as well, although it should be noted that this explanation of the additivity cannot apply to other examples.

However, this analysis, as it stands now, overgenerates. Notice, in particular, that the scalar presupposition that just is purported to trigger in (2) is very similar to what is standardly assumed for even (more on this in Section 3.2). If such a reading is available for just, then a reading of ( $8 \mathrm{a})$ is predicted that is be similar to the reading of ( $8 \mathrm{~b})$, but this reading does not seem to be available.

a. Just Patrick ${ }_{F}$ was in the room.

b. Even Patrick ${ }_{F}$ was in the room.

Specifically, let us assume as before that just in (8a) takes scope over the rest of the sentence. Also, suppose that the alternatives to Patrick are Andy, Bojan, and James. Then the $\mathrm{ONLY}_{2}$ reading of (8a) should have a scalar presupposition that it was relatively unlikely that Patrick was in the room, compared to these other people. However, this is intuitively what (8b) can mean, which suggests that the relevant scale of likelihood or unexpectedness could in principle be made contextually accessible, and (8a) cannot mean the same thing. Rather, (8a) only has an exclusivity reading, which entails that no one else is in the room. This problem therefore illustrates that Grosz's (2012) account at least needs to restrict the distribution of $\mathrm{ONLY}_{2}$, but it is not obvious to us how that can be done.

Another similar issue arises with a more ad hoc scale like the one used for (5). 
For just in this example, the following sentences are reasonable focus alternatives together with the scale where they are ordered in this order.
a. Patrick is a postdoc.
b. Patrick is an assistant professor.
c. Patrick is an associate professor.
d. Patrick is a full professor.

Note that this scale is not necessarily a scale of likelihood, because if Patrick is (known to be) in his 20s, for example, (9c) and (9d) are not very likely to be true, but (5) is still felicitous even in such a context. Rather, the relevant scale is perhaps that of academic ranks. But then, the same ordering of academic jobs should be able to order other sentences, for example, (10).
a. Patrick wrote a paper with a postdoc.
b. Patrick wrote a paper with an assistant professor.
c. Patrick wrote a paper with an associate professor.
d. Patrick wrote a paper with a full professor.

Consequently, the prediction is that a sentence like (11) should have a scalar reading based on the ordering in (10), but this does not seem to be the case. Rather, (11) only has an exclusivity reading.

Patrick wrote a paper just with a postdoc $F^{*}$

These issues show that Grosz's (2012) account, as it stands, is too liberal with respect to the distribution of the scalar reading, and it needs to somehow restrict the range of scales that just can access in yielding a scalar presupposition. However, in light of the above examples, it is not clear to us how such a constraint can be formulated. In particular, we do not see how this account can explain the contrast between (2) and (8) with respect to the availability of a minimal sufficiency reading.

\subsection{Coppock \& Beaver (2014)}

Coppock \& Beaver (2014) also attempt to account for the minimal sufficiency reading as a scalar reading of just, but unlike Grosz (2012), they attempt to do so with a general lexical entry for all uses of exclusive particles, given in (12), which is adapted from Coppock \& Beaver (2014: 394). For them, an exclusivity particle, represented here as ONLY, presupposes minimality and asserts maximality. Just like Grosz, they assume that a context specifies an order, $\geq$, among the contextually relevant focus alternatives, and CQ here refers to the set of sentences on the scale formed by $\geq$, which is taken to represent the current question under discussion.

$$
\begin{aligned}
& \llbracket \text { ONLY } S \rrbracket=\lambda w: \operatorname{MIN}_{\geq}(S)(w) . \operatorname{MAX}_{\geq}(S)(w) \\
& \text { a. } \quad \operatorname{MIN}_{\geq}(S)=\lambda w . \exists S^{\prime} \in \mathrm{CQ}\left[\llbracket S^{\prime} \rrbracket(w)=1 \text { and } S^{\prime} \geq S\right] \\
& \text { b. } \quad \operatorname{MAX}_{\geq}(S)=\lambda w . \forall S^{\prime} \in \mathrm{CQ}\left[\llbracket S^{\prime} \rrbracket(w)=1 \rightarrow S \geq S^{\prime}\right]
\end{aligned}
$$

In addition, Coppock \& Beaver assume that for the minimal sufficiency reading, just takes non-sentential scope. For example, for (2), repeated below, they would assume that just takes the DP, one cat, as its prejacent.

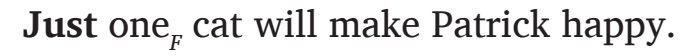


In order to give a full compositional account of an example like this, Coppock \& Beaver postulate some type-shifting operations, but we think it's not necessary to go into these technical details here (but note that we will make use of similar mechanisms in our account presented in the next section), as the more or less intuitive characterization of their analysis below is enough to understand the heart of the problem.

If the prejacent of just is one $e_{F}$ cat in (2), as Coppock \& Beaver assume, then, the focus alternatives will look like (13).
a. one cat
b. two cats
c. three cats

These alternatives can be order by something like remarkableness such that (13a) is the least remarkable member of the scale. With this scale, just triggers a presupposition that the prejacent one cat is low on this scale. Then the overall meaning of the sentence in (2) would be something along the lines of 'Being one cat is relatively unremarkable, and something that is one cat and no more than one cat will make Patrick happy'.

Note that it is crucial here that just is interpreted within the subject DP, because if the focus alternatives included the VP as well and looked like (7), then the scalar presuppose would be that it is unremarkable for one cat to make Patrick happy, which is intuitively the opposite of what we want to derive. Furthermore, since Coppock \& Beaver encode maximality in the semantics of just, so as to uniformly account for different uses of exclusive particles, the maximality in this case would amount to the negation of the focus alternatives formed with higher numerals. That is, it would negate that two or more cats will make Patrick happy, which is the opposite of the additivity observed with the minimal sufficiency reading of (2).

This analysis, however, also suffers from an overgeneration problem. The main issue comes from the crucial assumption that just takes the subject DP as its argument and introduces a scalar inference at this level. That is, because this assumption, it is predicted that the material in the VP should not matter for the availability of the minimal sufficiency reading, which is not borne out. More concretely, as already pointed out Section 1, (1a), repeated below, does not have a minimal sufficiency reading, and its only available reading is an exclusivity reading.

(1a) Just one ${ }_{F}$ cat is in the room.

The problem for Coppock \& Beaver is that the subject DP is exactly the same as in (2), so just should be able to make use of the same scale as above, in which case (1a) should be able to mean 'Something that is as unremarkable as one cat is in the room', without an exclusivity inference (so the sentence should be compatible with there being more than one cat), contrary to fact.

The same point can be made with the following pair of sentences: (3a) has a minimal sufficiency reading, while (14) does not.

Just a letter from her makes me happy.

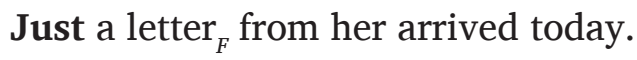

Thus, Coppock \& Beaver's account is simply unable to capture the fact that the VP portion of the sentence matters for the availability of minimal sufficiency readings, due to the crucial assumption that just takes scope at the DP-level. It seems to us that their analysis of the minimal sufficiency reading is hard to maintain. 


\section{Minimal sufficiency with even}

The problems for the previous analyses we discussed in the previous section suggest that in order to develop a more empirically adequate analysis of the minimal sufficiency reading, we have to understand its distribution better. To this end, we propose the following generalization: an overt even can be added felicitously to the examples that have minimal sufficiency readings, but not to the examples that don't have minimal sufficiency readings. This is illustrated by the following examples. Notice in particular the interpretive similarity between (2) and (4a), and (3a) and (15a).

a. Even just one ${ }_{F}$ cat will make Patrick happy.

b. \#Even just one ${ }_{F}$ cat is in the room.

a. Even just a letter from her makes me happy. $_{\text {b. \#Even just a letter from her arrived today. }}$

The same pattern is observed with the other examples of minimal sufficiency readings, (3b) and (3c), as the reader can verify.

It should also be remarked here that essentially the same meanings can be conveyed without just, as in (16), suggesting that an exclusive particle is actually not even a necessary ingredient to express the relevant scalar reading.

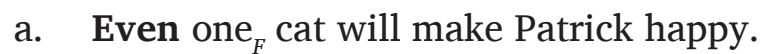

b. Even a letter ${ }_{F}$ from her makes me happy.

In light of these observations, we will take the following strategy in developing our account of the minimal sufficiency reading: We will first demonstrate how the readings of the felicitous examples containing overt even-i.e. (4a) and (15a), as well as (16) — can be accounted for with reasonable assumptions about the semantics of even and just.

Then we propose that our examples of minimal sufficiency readings, (2) and (3a), are to be understood in a parallel fashion to (4a) and (15a), by postulating a covert version of even, which we call 'covert even'. After that, in the next section, we will turn to the question of how to capture the infelicity of (4b) and (15b), and propose that our solution to this explains why (1a) and (14) do not have minimal sufficiency reading.

\subsection{The semantics of just}

Let us start with the semantics of just. The two previous analyses we discussed in the previous section assume that it can have a scalar reading, at least in sentences like (5). While this seems to be undeniable, the problems we pointed out for them, as well as the observations made earlier in this section, suggest that the minimal sufficiency reading might not be a special case of it. And at least in sentences like (4a) and (15a), which contain an overt even, the scalar component of the meaning seems to be coming from even, rather than from just. For this reason, we will analyze just in the relevant examples to be expressing an exclusivity reading, which can be characterized as in (17). ${ }^{3} \operatorname{Alt}(\phi)$ here is the contextually relevant set of focus alternatives to ' $\phi$ '.

\footnotetext{
${ }^{3}$ The truth of the prejacent of an exclusive particle is often analyzed as a presupposition, following Horn (1969) and Rooth (1992), among others. This is certainly an analytical possibility for us here, but as far as we can see, this would require no substantial change in our analysis to be presented below, although we suspect that it would complicate the presentation. Also, it should be pointed out that the presuppositionality of the prejacent is not uncontroversial in the literature (Beaver \& Clark 2008; Ippolito 2008; Coppock \& Beaver 2014; Panizza \& Chierchia 2019; Alxatib 2020).
} 
'Just $\phi$ ' asserts that ' $\phi$ ' is true and that all focus alternatives in $\operatorname{Alt}(\phi)$ that are not entailed by ' $\phi$ ' are false.

Note that for the minimal sufficiency reading of (4a), the prejacent of just should not be 'one ${ }_{F}$ cat will make Patrick happy', because the exclusive reading in that case would entail two cats will not make Patrick happy. We therefore assume that just should take scope right above or within the subject DP. This assumption is similar to Coppock \& Beaver's (2014) but we are crucially assuming that just has an exclusivity reading here, rather than a scalar reading.

In fact, such a DP-level reading of the exclusivity reading of just is independently observed. Consider (18), for example, which is ambiguous with respect to the scope of the exclusivity inference of just.

\section{Just this teapot ${ }_{F}$ costs $£ 3$.}

If the exclusivity inference of just is understood at the sentential scope, the example will entail that nothing other than the relevant teapot costs $£ 3$. But this example has another reading, according to which buying that teapot alone without anything else will cost $£ 3$. This latter reading, unlike the former reading, is compatible with another item-for example, a milk jar-costing $£ 3$ as well. It should also be noted that this reading is not a minimal sufficiency reading, as it doesn't have a scalar component (although it could also have a minimal sufficiency reading; we will come back to this point in Section 5). Rather it is a kind of exclusivity reading in the sense that it is excluding other items from the total price. ${ }^{4}$

In order to work out the composition of the DP-level exclusivity inference of just, we will type-generalize the meaning given in (17) above. This can easily be done following the standard recipe (as is done in Rooth 1985, for example). Specifically, we define conjoinable types as follows.

Conjoinable types

a. $t$ is a conjoinable type.

b. If $\sigma$ is a type and $\tau$ is conjoinable type, then $\langle\sigma, \tau\rangle$ is a conjoinable type.

c. Nothing else is a conjoinable type.

Then we define generalized entailment, generalized conjunction, and generalized negation as follows.

(20) Generalized entailment $(\Rightarrow)$ : Let $x$ and $y$ be of the same conjoinable type.

a. If $x, y \in D_{t}$, then $x \Rightarrow y$ is the case iff $x=0$ or $y=1$.

b. If $x, y \in D_{\langle\sigma, \tau\rangle}$, then $x \Rightarrow y=\lambda z \in D_{\sigma} . x(z) \Rightarrow y(z)$.

(21) Generalized conjunction ( $\Pi$ ): Let $x$ and $y$ be of the same conjoinable type.

a. If $x, y \in D_{t}$, then $x \sqcap y$ is the case iff $x=y=1$.

b. If $x, y \in D_{\langle\sigma, \tau\rangle}$, then $x \sqcap y=\lambda z \in D_{\sigma} \cdot x(z) \sqcap y(z)$.

(22) Generalized negation ( $\sim$ ): Let $x$ be of a conjoinable type.

a. If $x \in D_{t}$, then $\sim x$ is the case iff $x=0$.

b. If $\mathrm{x} \in D_{\langle\sigma, \tau)}$, then $\sim x=\lambda z \in D_{\sigma} . \sim x(z)$.

\footnotetext{
${ }^{4}$ This DP-level reading seems to have an inference that buying something else along with the teapot will cost more than $£ 3$, but this inference seems to be defeasible and is likely to be a conversational implicature. We will not try to analyze this implicature in this paper.
} 
Using these notions, the type-generalized version of (17) can be stated as (23). ${ }^{5}$ For a set $S$ whose members are of the same conjoinable type, we write $\Pi S$ for the grand generalized conjunction of the members of $S$.

$$
\llbracket \text { just } \alpha \rrbracket=\llbracket \alpha \rrbracket \sqcap \Pi\{\sim \llbracket \beta \rrbracket \mid \beta \in \operatorname{Alt}(\alpha) \text { and it is not the case that } \llbracket \alpha \rrbracket \Rightarrow \llbracket \beta \rrbracket\}
$$

Using this semantics, the sentential-level exclusivity inference of (18) is derived as follows. In fact, there are two ways of deriving it. One is by assuming that just simply takes sentential scope (potentially via covert movement or some other scope taking mechanism). The other way is to assume that the subject DP this teapot denotes a generalized quantifier via the usual Lift operation that turns an entity into a generalized quantifier, i.e. $a \mapsto \lambda P . P(a)=1$, and just applies to this generalized quantifier, turning it to the generalized quantifier that is true of any predicate that is true of the relevant teapot and nothing else, i.e. $\lambda P . P(t)=1$ and for other relevant entities $x, P(x)=1$, where $t$ is the relevant teapot. This latter derivation is important for later discussion in this paper, and should be emphasized at this point: even if just is syntactically at the DP-level, the exclusivity inference can arise at the sentential level.

In order to derive the DP-level exclusivity reading of just in (18), we have to make assumptions about plurality, because intuitively, what is being excluded here is the possibility that the subject denotes a plural entity. To this end, we will adopt Winter's (2001) approach to plurality (see also Champollion 2016), which allows us to straightforwardly use the type-general semantics of just given in (23). ${ }^{6}$

Winter (2001) represents plural entities as sets of entities and treats predicates such as costs $£ 3$ as true of such pluralities qua sets of entities (in this case including singleton sets), and we will follow him here, although this assumption is not crucial for us. What is important is that he proposes a way to account for the non-distributive reading of conjoined proper names like Anna and Becky with generalized conjunction $\square$ (rather than a separate plurality forming conjunction). The derivation for this example goes as follows. First we lift the two individuals to quantifiers, and conjoining them via $\Pi$, which results in the distributive generalized quantifier, $\lambda P . P(a)=1$ and $P(b)=1$, where $a$ is the denotation of Anna and $b$ is the denotation of Becky. To get the non-distributive reading out of this, the minimality-operator (min-operator) is applied to this generalized quantifier, which yields a predicate of predicates that is only true of the minimal ones with respect to $\Rightarrow$ among the predicates that this generalized quantifier is true of, i.e. $\min (Q):=\lambda P . Q(P)=1$ and there is no $P^{\prime}$ such that $Q\left(P^{\prime}\right)=1$ and $P^{\prime} \Rightarrow P$. For the example at hand, it will be a predicate that is only true of the predicate that is only true of $a$

\footnotetext{
${ }^{5}$ We could incorporate the notion of Innocent Exclusion due to Fox (2007) in the semantics of just, as follows.
}

(i) $\quad \llbracket$ just $\alpha \rrbracket=\llbracket \alpha \rrbracket \Pi \Pi\{\sim \llbracket \beta \rrbracket \mid \beta \in \operatorname{IE}(\alpha)\}$

a. $\operatorname{IE}(\alpha)=\bigcap\{S \mid S$ is a maximal excludable set of alternatives of $\alpha\}$

b. $S \subseteq \operatorname{Alt}(\alpha)$ is an excludable set of alternatives of $\alpha$ iff $\llbracket \alpha \rrbracket \sqcap \Pi\{\sim \llbracket \beta \rrbracket \mid \beta \in S\} \neq \perp$

(ii) Generalized contradiction $(\perp)$ : Let $x$ be of a conjoinable type.

a. If $x \in D_{t}$, then $x=\perp$ iff $x=0$.

b. If $x \in D_{\langle\sigma, \tau}$, then $x=\perp$ iff for each $\mathrm{z} \in D_{\sigma}, x(z)=\perp$.

This alternative formulation would circumvent certain empirical issues that could arise for (23), which are related to the so-called symmetry problem for scalar implicatures, but none of our examples in this paper give rise to such issues. See Fox \& Katzir (2011) for detailed discussion.

${ }^{6}$ We leave open the possibility of using other theories of plurality, especially those that make use of a plurality forming conjunction (sometimes called 'non-Boolean conjunction'), in addition to $\Pi$. It should be remarked, however, that in Section 4, we will make crucial use of one aspect of Winter's (2001) theory that a collective interpretation requires additional mechanisms (see below), in order to account for the distribution of the DP-level exclusivity reading of just. 
and $b$, i.e. $\lambda$ P. $P=[\lambda x, x=a$ or $x=b]$. Then existential type-shift applies to this set of predicates turning it into a plural existential quantifier, i.e. $\mathcal{P} \mapsto \lambda Q . \exists X \mathcal{P}(\chi(X))=1$ and $Q(X)=1$, where $\chi(X)$ is the characteristic function of the set $X$. This can now combine with a non-distributive predicate which takes a set of entities as an argument. We will henceforth call the combination of the two operations, the min-operator + the existential type-shift, the plurality-forming type-shift.

Assuming this theory of plurality, we can account for the DP-level exclusivity reading of just in (18) as follows. First, just applies to the lifted version of the individual denoted by this teapot, which yields the generalized quantifier $\lambda P . P(t)=1$ and there's no other relevant entity $x$ such that $P(x)=1$, where $t$ is the entity that this teapot refers to and $x$ ranges over the referents of its focus alternatives. Then via the plurality-forming typeshift, we obtain the plural quantifier: $\lambda Q . Q(\{t\})=1$. This captures the reading of (18) that we are after.

With this analysis of just at hand, let us now come back to (4a), repeated here.

Even just one $_{F}$ cat will make Patrick happy.

As explained above, just here should trigger an exclusivity inference at the DP-level. To derive it, we have to make assumptions about the semantics of one cat and its focus alternatives. We assume that DPs with bare numerals like one cat have existential meanings. In many examples such DPs receive a maximal, bilateral ('exactly') reading, which could be captured by analyzing the denotation of one cat as (24a) and that of two cats as (24b).

$$
\begin{aligned}
& \text { a. } \lambda P . \exists ! x[x \text { is a cat and } P(x)=1] \\
& \text { b. } \lambda P . \exists ! X[X=\{x, y\} \text { and } x \text { and } y \text { are distinct cats and } P(x)=P(y)=1]
\end{aligned}
$$

However, it has been debated in the literature whether the maximality of bare numerals should be accounted for semantically like this, or it should be derived as a scalar implicature from non-maximal, lower-bounded ('at least') readings of bare numerals as in (25) (cf. Horn 1972; Geurts 2006; Breheny 2008; Spector 2013). ${ }^{7}$

$$
\begin{array}{ll}
\text { a. } & \lambda P . \exists x[x \text { is a cat and } P(x)=1] \\
\text { b. } & \lambda P . \exists X[X=\{x, y\} \text { and } x \text { and } y \text { are distinct cats and } P(x)=P(y)=1]
\end{array}
$$

Notice that if DPs with bare numerals receive bilateral readings like (24), the contribution of just would be vacuous, because, for example, (24a) and (24b) are already incompatible with each other (in the sense of generalized contradiction, $\perp$, as defined in footnote 5). As a vacuous use of just seems to be generally infelicitous, let us assume the lower-bounded semantics of numerals given in (25) here. In fact, it seems that all accounts of bare numerals assume that the lower-bounded semantics is at least available, if not by default. Then, just one cat will denote the generalized quantifier in (24a). This can further undergo the plurality-forming type-shift, yielding the plural quantifier, $\lambda Q . \exists X[X$ is a singleton set containing one cat and $Q(X)=1] .^{8}$

\footnotetext{
${ }^{7}$ We could give an inherently plural meaning to two cats, $\lambda Q . \exists x \exists y[x$ and $y$ are distinct cats and $Q(x, y)=1]$, but this can be derived by the plurality-forming type-shift from (25b) (Winter 2001; Champollion 2016).

${ }^{8}$ Note that the semantic contribution of just is vacuous at this point, as the exact same meaning will be expressed without just, regardless of whether the bare numeral receives a lower-bounded or bilateral reading. A similar remark applies to the DP-scope exclusivity reading of (18) above. Let us assume that just is still licensed in such cases, because its truth-conditional contribution is locally non-trivial. See Mayr \& Romoli (2016); Meyer (2016); Sudo (2017), and references therein for relevant discussion. Also, note that this triviality is about the truth-conditional aspect of the meaning, and just could other interpretive effects, as we will discuss in Section 5.
} 
The DP-level exclusivity reading of just in (15a) can be analyzed analogously, but to save space, we will not spell it out here.

(15a) Even just a letter ${ }_{F}$ from her makes me happy.

Having analyzed the semantic contribution of just in (4a) and (15a), we will now turn to the semantics of even.

\subsection{The semantics of even}

Even is standardly analyzed as a focus sensitive particle that introduces two presuppositions based on focus alternatives, a scalar presupposition and an additive presupposition (Karttunen \& Peters 1979; Rooth 1985; Kay 1990; Wilkinson 1996; Crnič 2011). To illustrate, consider (26).

$$
\text { Even } \text { Marten }_{F} \text { danced. }
$$

This sentence has a scalar presupposition that Marten was unlikely to dance, and also an additive presupposition that someone else also danced. Since these inferences are absent from the version of the sentence without even, they should arise from even. We can formalize the semantic contribution of even as follows.

'Even $\phi$ ' presupposes:

a. that $\phi$ is relatively unlikely to be true among $\operatorname{Alt}(\phi)$; and

b. that there is $\psi \in \operatorname{Alt}(\phi)$ that is not entailed by $\phi$ and is true.

Two caveats are in order here. Firstly, proposals in the literature differ as to how exactly the scalar presupposition in (27a) is characterized, e.g. in terms of likelihood, noteworthiness, or something more general (Rooth 1985; Kay 1990; Herburger 2000; Greenberg 2018). We are certainly not in a position to solve this debate, and we will try to make sure that our analysis of the minimal sufficiency reading does not crucially hinge on one particular view on this point. Secondly, it is known that the additive presupposition (27b) is not always observed, which has led some to propose that it is not inherent to the semantics of even, but as far as we can see, there is no theory at the moment that can fully explain when exactly the additive presupposition arises (see Rullmann 1997; 2007 and Crnič 2011 for attempts). For this reason, we will stick to the above 'classical analysis', where it is encoded in the semantics of even.

Now, to see how the above semantics works, let us apply it to (26) above. The focus

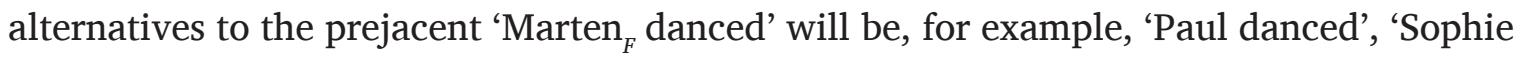
danced', 'Sascha danced', etc. The scalar presupposition amounts to that it was relatively unlikely among these alternatives that Marten would dance and the additive presupposition to that at least one more person danced, which capture the observed reading of (26). Let us now come back to (4a).

(4a) Even just one ${ }_{F}$ cat will make Patrick happy.

As discussed above, the exclusivity inference of just takes DP-level scope in this example. For even, we assume that it takes sentential scope for the following reason. Recall at this point the problem for Coppock \& Beaver (2014). Under their account of the minimal sufficiency reading, the scalar meaning comes from just, which takes a DP-level scope, and for that reason it is oblivious to the material outside the local DP. But that was shown to result in an overgeneration problem, because the VP portion of the sentence matters for 
the availability of a minimal sufficiency reading. The sentence in (4a) contains an overt scalar particle even, but if it introduces a scalar inference at the level of the subject DP, then it will run into essentially the same problem. That is, the contrast between (4a) and (4b) will be difficult to explain.

$$
\text { \#Even just one }{ }_{F} \text { cat is in the room. }
$$

For this reason, it is crucial that even somehow sees the VP material as well. The most natural way is to assume that it takes sentential scope here. Note that we do not make particular commitments about the syntax of these sentences. It is possible that even is structurally located within the DP on the surface or it in fact sits above the rest of the sentence. In the former case, even would have to take wider scope than its syntactic position, which could be done with a standard mechanism of scope shift (e.g. Quantifier Raising, or type-shift).

Now, notice that (4a) contains two focus sensitive particles, even and just, but only one focused material, one. We have to say something about how this works, given that Rooth (1992) and many later studies assume that when a focus operator operates on a set of focus alternatives, it 'eats up' these focus alternatives, and yields a trivial set of focus alternatives as a consequence. More concretely, for a phrase like 'just [one ${ }_{F}$ cat]', the relevant focus alternatives that just operations on will be a contextually determined subset of $\{$ ' $\xi$ cat' $\mid$ ' $\xi$ ' is a numeral\}, but once the meaning of just is computed, a higher phrase cannot refer to these focus alternatives anymore. Thus, it is often assumed that in a configuration like 'even [just one ${ }_{F}$ cat will make Patrick happy]', the set of focus alternatives for even is just a singleton set containing 'just one ${ }_{F}$ cat will make Patrick happy'. Of course, if that is the case, then the semantic contribution of even will be vacuous, which needs to be avoided this situation. We will propose a simple solution to this issue in the next subsection and show that the intended reading of (4a) can be derived together with the semantics of just and even introduced above.

\subsection{Two focus particles and one focused item}

Rooth (1992) introduces the assumption that focus particles eat up focus alternatives, but he does so without presenting empirical evidence for it. ${ }^{9}$ We would like to question this assumption here. In fact, empirical facts independent from minimal sufficiency readings suggest that a single focussed phrase can actually associate with multiple focus sensitive operators, at least on the surface. Such examples are discussed and analyzed by Krifka (1991). Consider the following example taken from Krifka (1991: 131), for example.

At yesterday's party, people stayed with their first choice of drink. Bill only drank WINE, Sue only drank BEER, and John even only $_{2}$ drank [WATER $]_{1,2}$

Krifka's analysis of this example is couched in the Structured Meaning approach to focus. We could state our analysis in this alternative approach, but we stick to the Roothian approach we have been assuming here. Recasting Krifka's idea for such complex foci in a way that is compatible with our framework of choice amounts to dropping Rooth's aforementioned assumption, and assume instead that focus alternatives can (at least optionally) remain active after the structurally lower focus operator has operated on

\footnotetext{
${ }^{9}$ Rooth (1992: 94) simply states as follows: "in the expression $\phi \sim v$, focus has been interpreted, so we want to neutral the semantic effect of the foci in $\phi$ ".
} 
them. ${ }^{10}$ With this revised assumption, the set of focus alternatives for only in (28) will be a subset of $\left\{\right.$ 'drank $\xi^{\prime} \mid ' \xi$ ' = 'water', 'beer', 'wine', 'vodka', ...\}, and the set of focus alternatives for even will be a subset of \{'only drank $\xi$ '|' $\xi$ ' = 'water', 'beer', 'wine', 'vodka', ...\}. Consequently, the scalar inference that even yields is that (John's) drinking water but nothing else is less likely/more remarkable than (John's) drinking beer and nothing else, (John's) drinking wine and nothing else, etc., which captures the intended reading of (28).

We therefore think that there are enough empirical reason to assume that focus alternatives do not necessarily become inactive after a focus operator has operated on them. Now we have all the ingredients to analyze (4a). The focus alternatives for just are, as before, a subset of $\{$ ' $\xi$ cat'|' $\xi$ ' is a numeral $\}$. On the other hand, the focus alternatives for even, which we assume takes sentential scope here, will be sentences like the following:
a. Just one $e_{F}$ cat will make Patrick happy.
b. Just two ${ }_{F}$ cats will make Patrick happy.
c. Just three ${ }_{F}$ cats will make Patrick happy.

There can well be more focus alternatives, but without loss of generality we will just use these three here. Note that the predicate will make Patrick happy is a non-distributive predicate, so we assume that the subject DPs in these sentences undergo the pluralityforming type-shift. Crucially, we assume that the plurality-forming type-shift applies after the exclusivity inference is introduced by just, because otherwise the exclusivity inference would take scope at the sentential level (see the discussion in Section 3.2). Consequently, (29a) means that if there is an entity made up of exactly one cat, that entity will make Patrick happy, (29b) means that if there is a plural entity made up of exactly two cats, that plural entity will make Patrick happy, and likewise (29c) means that if there is a plural entity made up of exactly three cats, that plural entity will make Patrick happy. ${ }^{11}$

With these focus alternatives, the scalar presupposition of even will be that (29a) is relatively unlikely/more remarkable among them, and its additive presupposition will be that at least one more focus alternative is true. These seem to capture the intuitive reading of (4a).

As remarked in passing at the end of Section 3.1, the truth-conditional contribution of just in (4a) is locally meaningful (with the lower-bounded semantics for bare numerals), but truth- conditionally vacuous in the end, due to the plurality-forming type-shift (although it could have other interpretive effects; see Section 5). This leads to a prediction that just is not a necessary component for this reading, which is in fact borne out: As we saw at the beginning of the present section, (16a) has essentially the same reading as (4a).

(16a) Even one ${ }_{F}$ cat will make Patrick happy.

Our analysis for this sentence proceeds as follows. Here, there is only one focus sensitive operator, even, and the relevant alternatives include the sentences in (30).

\footnotetext{
${ }^{10}$ Do focus alternatives ever become inactive? For sentences like 'JOHN ${ }_{F}$ only drank WATER', it might be more desirable to say that the focus alternatives to water become inactive, as the focus on water does not (need to) behave like a free focus, unlike the one on John. Then, perhaps focus alternatives only optionally become inactive.

${ }^{11}$ An anonymous reviewer asks whether the de re/de dicto ambiguity in this example matters for the availability of the minimal sufficiency reading. Given its genericity, it is more natural to read cat here de dicto in this particular case, but we do not think this is relevant. In fact, a similar reading is clearly available even when

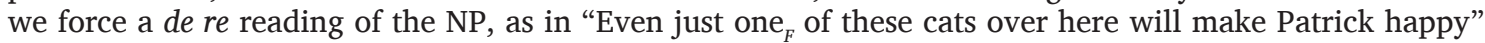
(although, strictly speaking, in this example the de re and the de dicto reading collapse to one; in a generic statement like this, it is generally not possible to single out the de re reading). Generally, we do not see a correlation with de re/de dicto and minimal sufficiency readings.
} 

a. One cat will make Patrick happy.
b. Two cats will make Patrick happy.
c. Three cats will make Patrick happy.

As discussed in Section 3.1, bare numerals can have lower-bounded readings or bilateral readings, but as before, the existential quantifier embedded in the plurality-forming typeshift will neutralize their distinction at the global level, and all the sentences in (30) will effectively have lower-bounded ('at least') readings. ${ }^{12}$ Then the presuppositions that even yields will be exactly the same as in the previous example. That is, the scalar presupposition is that (30a) is less likely/more remarkable than the other alternatives, and the additive presupposition is that at least one more alternative is true.

The other example containing both even and just, namely (15a), and its variant without just, (16b), can be analyzed analogously, although more contextual information is needed to determine the relevant set of focus alternatives in these cases.

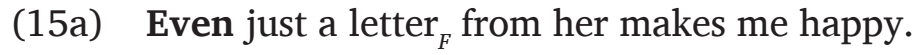

(16b) Even a letter from her makes me happy.

For the sake of illustration, let us consider the following focus alternatives for even. Just will be included for (15a) but not for (16b):

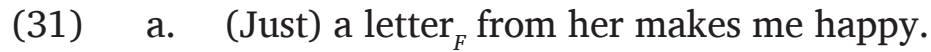

b. (Just) a phone call from her makes me happy.

c. (Just) a video call ${ }_{F}$ from her makes me happy.

We again assume that just introduces an exclusivity inference at the DP-level and since the predicate is collective, the whole DP undergoes the plurality-forming type-shift, due to which the exclusivity inference is neutralized, as in the previous examples. Consequently, each sentence in (31) has the same truth-conditions with or without just. Now, the scalar presupposition of even amounts to that (31a) is relatively unlikely/remarkable, and the additive presupposition to that at least one of (31b) and (31c) is also true, which capture the observed readings of (15a) and (16b).

\subsection{Minimal sufficiency with covert even}

Having explained how the examples that contain both an overt even and just can be analyzed, we are now ready to account for our initial examples, (2) and (3a), which do not contain an overt even. We simply propose that these sentences contain a covert version of even, which we assume has the same semantics as its overt counterpart (cf. Chierchia 2013). Then they can be analyzed in a completely parallel fashion to what we have just demonstrated above.

We furthermore would like to convince the reader that it is not too far-fetched to postulate a covert version of even. A growing body of research, at least since Groenendijk \& Stokhof (1984), suggests that there is a mechanism that gives rise to an exclusivity inference without an overt exclusive particle, although there is a heated debate about the nature of that mechanism in the current theoretical literature, especially with respect to whether or not the mechanism is purely pragmatic or something that is present at the syntax-semantics interface (see, for example, Chierchia et al. 2012, Geurts 2010, and references therein).

\footnotetext{
${ }^{12}$ There could be a sentence-level exclusivity inference on top of this, which is potentially a scalar implicature, but this is irrelevant here.
} 
Whatever the true nature of the mechanism, exclusivity interpretations are clearly available for sentences like the following, despite the fact that they do not contain an exclusive particle.
a. Who danced? - Marten $_{F}$ did.
b. How many people danced? - Ten ${ }_{F}$ people did.

We need not go into the theoretical debate about exclusivity inferences here (see the references above), but what is relevant for our purposes is Chierchia's (2013) idea that a similar mechanism exists that gives rise to scalar inferences. ${ }^{13}$ To illustrate this, Chierchia (2013: 109) mentions the following example.

$$
\text { Really everybody came to my party. Imagine that my } \mathrm{ex}_{F} \text { came. }
$$

Here, the second sentence can be paraphrased by 'Imagine that even my ex $_{F}$ came', and has a scalar inference that is similar in nature to the scalar inference of even.

Parallel to the debate on exclusivity inferences mentioned above, the question then arises as to whether such scalar inferences without an over scalar particle should be understood in terms of some pragmatic reasoning or in terms of a covert operator that is present at the syntax-semantics interface.

In this connection, it should be noted that previous studies on exclusivity inferences have raised a number of examples that seem to involve an exhaustivity inference below some linguistic operator, which have been used to argue for the operator-based view, and simultaneously against the pragmatic view of exclusivity inferences. However, whether or not these examples are actually problematic for a pragmatic analysis has been highly controversial (see, for example, Sauerland 2004; Russell 2006; Geurts 2010). Importantly for us, similar examples of embedded scalar inferences can be constructed, as an anonymous reviewer pointed out to us. For instance, the second sentence of (33) seems to involve the scalar inference under imagine, and also, more importantly for our interests here, the following sentence seems to have a minimal sufficiency reading under the scope of doubt.

Andy doubts that just one ${ }_{F}$ cat will make Patrick happy.

If one assumes that covert even is a pragmatic mechanism of some sort, one will certainly need an analysis of how it can take scope below operators like doubt.

While acknowledging the importance of this observation, however, we cannot evaluate the feasibility of the pragmatic view with respect to such sentences, in the absence of an explicitly formulated pragmatic theory of scalar inferences. In fact, it is not necessary to assume that a pragmatic mechanism cannot affect the semantics locally within the scope of an operator like doubt, even for exclusivity inferences (although such an assumption is certainly reasonable for purely Gricean conversational implicatures; cf. Geurts's 2010 discussion of pragmatic enrichment of lexical meanings). For this reason, we are reluctant to reject the pragmatic view altogether at this point. But as far as the present paper is concerned, the only crucial assumption is that such a mechanism exists (and can interact with linguistic operators), and we will not decide whether that is done via a covert operator in the syntax/semantics, or via some pragmatic mechanism. That being said, for the ease of exposition, we will speak for the rest of this paper as if the relevant mechanism is a silent operator that has the same semantics as even, and call it 'covert even'.

\footnotetext{
${ }^{13}$ Also relevant here are works on NPI licensing in terms of such a mechanism (Lahiri 1998; Nakanishi 2006;
} Guerzoni 2003; Chierchia 2013). 


\section{Blocking minimal sufficiency readings}

We have just demonstrated that sentences like (4a) and (16a) that contain both overt even and just and have readings similar to our primary examples of the minimal sufficiency readings, (2) and (3a), can be analyzed using the more or less standard semantic analyses of even and just with some reasonable auxiliary assumptions. We then propose to analyze (2) and (3a) in the same way by postulating a covert variant of even.

Importantly, however, that we can derive the minimal sufficiency readings for (2) and (3a) is not in itself an advantage of our analysis over the two previous analyses discussed in Section 2. Rather, as stressed there, we also want to explain when a minimal sufficiency reading is not available. For example, we want to understand why (1a) and (14), repeated here, do not have minimal sufficiency readings.

(1a) Just one $e_{F}$ cat is in the room.

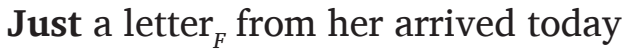

Our strategy to account for the lack of minimal sufficiency readings in these examples is, again, by resorting to covert even. As we have already observed, these sentences are incompatible with an overt even, as shown in (4b) and (16b), respectively. Given that under our analysis covert even is a necessary ingredient of the minimal sufficiency reading, if we understand why (4b) and (16b) are infelicitous, we can also straightforwardly account for the lack of minimal sufficiency readings in (1a) and (14) by resorting to the fact that they are simply incompatible with covert even. In this section, we will discuss how this can be done.

Let us start with (4b). Parallel to our analysis for (4a), the focus alternatives for even in (4b) should look like (35).
a. Just one $e_{F}$ cat is in the room.
b. Just $\mathrm{two}_{F}$ cats are in the room.
c. Just three ${ }_{F}$ cats are in the room.

These sentences are naturally read as involving an exclusivity inference at the sentential level. That is, (35a) entails that there is no more than one cat in the room, and similarly for (35b) and (35c).

From these alternatives, even triggers a scalar presupposition to the effect that (35a) is less likely/more remarkable than the others. This could be the satisfied, if, for whatever reason, multiple cats are expected in the room. However, the additive presupposition of even will be problematic here, because the alternatives in (35) are logically mutually incompatible, due to the exclusivity inferences, meaning no more than one of them can be true at a time. We could make use of this fact to explain why (4b) is unacceptable.

However, notice that we are assuming that the exclusivity inferences take scope at the sentential level in these focus alternatives. This is arguably the only intuitively available interpretations of (35). (35a), for example, cannot mean that there is $x$ such that $x$ consists of exactly one cat and $x$ is in the room, which would be compatible with there being more than one cat in the room. One might think that this is because is/are in the room is a distributive predicate, so just one $e_{F}$ cat cannot undergo the plurality-forming type-shift, and in that case, even if just takes scope within the DP, the exclusivity inference still takes scope at the sentential level, as explained in Section 3.1.

However, under Winter's (2001) theory, which we are adopting here, there is no reason why the plurality-forming type-shifts cannot apply when the predicate is distributive, 
because distributive predicates are compatible with plurality denoting expressions like these cats, as in 'These cats are in the room'. One way to understand this is that each distributive predicate has a version that can apply to plural entities (more on this below). It is then theoretically possible to apply the plurality-forming type-shift to just one $e_{F}$ cat, and apply the result to the plural version of the predicate is in the room. Importantly, if this were possible, then the focus alternatives in (35) would end up having lowerbounded readings without exclusivity inferences, because of the existential quantification embedded in the plurality-forming type-shift. Then they would be compatible with each other, so the additive presupposition would no longer be conflicting, and we would fail to explain the infelicity of (4b).

But how about the scalar presupposition? Notice that these focus alternatives with the lower- bounded readings would be ordered by entailment, with (35c) asymmetrically entailing (35b), which in turn would be asymmetrically entailing (35a). Then, logically, (35a) would be the most likely alternative. This means that if the scalar presupposition of even is about likelihood, then it would become contradictory, which might look useful in explaining the infelicity of (4b). That is, according to this idea, when the exclusivity inference of just is nullified by the plurality-forming types-shift, the scalar presupposition becomes problematic, while when it takes sentential scope, the additive presupposition becomes problematic. Thus, either way, the example is rendered infelicitous.

However, we think this explanation is wanting for several reasons. For one, the reasoning in the previous paragraph crucially hinges on the likelihood-based analysis for the scalar presupposition of even, more specifically, on the monotonicity of likelihood with respect to entailment, i.e. if $p$ entails $q$, then $\Pi(p) \leq \Pi(q)$, where $\Pi$ is a probability distribution over propositions. As mentioned in Section 3.2, there is currently no consensus on how the scalar presupposition of even should be characterized. In fact, a number of examples have been raised that seem to be problematic for the likelihood-based analysis, and alternative proposals have been put forward that make use of 'noteworthiness' or some other gradable notion, which is not necessarily monotonic with respect to entailment (see Herburger 2000 and Greenberg 2018, for example).

Secondly, and more crucially, an explanation analogous to the one above is simply unavailable for the other infelicitous example we are after, namely (16b), repeated here.

(16b) Even a letter ${ }_{F}$ from her makes me happy.

To see this, let's consider the following focus alternatives for even in this sentence.
a. Just a letter ${ }_{F}$ from her arrived today.
b. Just a phone call from her arrived today.

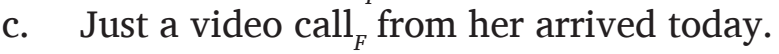

As before, if just introduces an exclusivity inference at the sentential level in these focus alternatives, the additive presupposition of even will be inconsistent with the prejacent. However, this time, the presuppositions derived from (36) with the DP-level exclusivity inferences will be consistent. That is, in this case, (36a) will mean that there is an $x$ such that $x$ consists of nothing but a letter from her and $x$ arrived, and similarly for (36b) and (36c). Then the alternatives are mutually consistent, so the additive presupposition will not be an issue. Furthermore, one could imagine a context that satisfies the scalar presupposition. For instance, in a modern society, it is much less likely to receive a letter than to receive a phone call or a video call. Consequently, the prediction is that (16b) should be felicitous, contrary to fact. 
We think what needs to be explained here is why sentences like (35) and (36) have to have an exclusivity inference at the sentential level, unlike the focus alternatives for even in sentences like like (4a) and (15a).

(4a) Even just one ${ }_{F}$ cat will make Patrick happy.

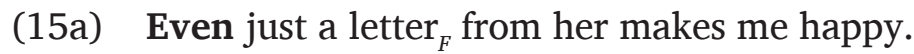

Since the subject DP is the same between (4a) vs. (4b) and (15a) vs. (15b), the crucial difference should reside in the other part of each sentence. These pairs of examples differ in many ways. For example, it might look as if genericity is a factor. That is, (4a) and (16a) are generic statements, while (4b) and (16b) are episodic statements. However, notice that our first example of a DP-level exclusivity reading of just, (18), is not generic. Furthermore, (18) is compatible with even, as in (37).

Even just this teapot costs $_{3}$.

Note that in a context where $£ 3$ is cheap, (37) is not felicitous (we will come back to this observation in the next section). Importantly, even in such a context, (18) is felicitous, suggesting that the compatibility with overt or covert even is not a necessary condition for the DP-level exclusivity reading of just.

We claim that a primary factor that determines whether the DP-level reading of just is available or not is distributivity: predicates like is/are in the room and arrived today are distributive, while predicates used in examples with DP-level exclusivity inferences, will make Patrick happy, makes me happy, and costs £3, are non-distributive. With this in mind, we propose that the exclusivity inference of just cannot take DP-level scope if the predicate the DP combines with is distributive.

One way to understand this restriction is by assuming that the plurality-forming typeshift is triggered by the predicate and is not encoded in the DP. In the previous section, we followed Winter's (2001) in assuming that a generalized quantifier is type-shifted via the plurality-forming type-shift to yield a non-distributive interpretation of the quantifier. Suppose instead that this type-shift is actually encoded as part of the meaning of the VP. Then, the denotation of the subject just this teapot in the examples in (38), for example, will be identical regardless of the nature of the predicate.

a. Just this teapot costs $£ 3$.

b. Just this teapot is in the room.

That is, in both examples, it denotes the following distributive generalized quantifier: $\lambda P$. $P(t)=1$ and for all other relevant entities $x, P(x)=0$, where $t$ is the denotation of the teapot. A non-distributive VP like costs $£ 3$ then applies the plurality-forming type-shift to this generalized quantifier, before feeding it the function $\lambda X . X$ costs $£ 3$. This captures the DP-level exclusivity inference for (38a). By contrast, for (38b), the exclusivity inference must take sentential scope, simply because this sentence does not involve the pluralityforming type-shift.

Recall at this point that (38a) is ambiguous and can also have a sentential scope exclusivity reading. To derive it, we could assume that just can take scope at the sentential level in this example, so the alternatives it operates on includes the predicate. Alternatively, we could analyze it by letting the entire subject DP, just this teapot, take scope over the rest of the sentence. Then the sentence will be true iff $c$ is the only relevant thing $x$ such that $\{x\}$ costs $£ 3$. In other words, as long as the exclusivity takes scope of the plurality-forming type-shift, the sentential scope exclusivity reading ensues. 
Let us tied up some loose ends. Obviously, some DPs can denote pluralities without typeshifting, e.g. these teapots. Such DPs can combine with distributive predicates, as in 'These teapots are in the room'. The standard way to analyze such sentences is by postulating a covert distributivity operator (or a type-shifting rule that amounts to the same thing), which applies to the VP and extracts singular entities from its plural argument (see Roberts 1987; Schwarzschild 1996; Winter 2001; Champollion 2017, among many others): $\lambda P$. $\lambda X$. for each $x \in X, P(x)=1$. We would like to point out in this connection that the pluralityforming type-shift can be seen in a similar way. Notice, in particular, that for predicates like cost $£ 3$, inherently plural DPs like these teapots give rise to ambiguity: It can be read collectively, meaning the teapots together cost $£ 3$, or distributively, meaning each of them costs $£ 3$. The latter distributive reading can be derived with the covert distributivity operator (strictly speaking, plurality formation $a \mapsto\{a\}$ is also necessary). The former, collective reading of 'These teapots cost $£ 3$ ', on the other hand, shows that predicates like costs $£ 3$ do not always involve the plurality-forming type-shift. This ambiguity of a collective predicate as to whether or not it involves the plurality-forming type-shift might seem to be conceptually unwelcome, but we would like to draw an analogy with the distributivity operator here. That is, the reason why a covert distributivity operator is postulated as a covert operator (or as a type-shifting rule) is because it applies optionally. If not, it could be integrated in the semantics of the predicate. We could see the mechanism for constructing plural entities is of a similar nature, i.e. as a covert operator that is optionally present.

One might be wondering why Winter (2001) encoded the plurality-forming type-shift in the DP, rather than in the predicate, to begin with. This is because of examples like (39), which Winter argues involves plurality formation within one of the conjuncts.

\section{Andy and Andy and Patrick wrote a paper.}

If the conjunctions here were simply generalized conjunctions, then the subject DP of this example should denote the same thing as Andy and Patrick. We think, however, that it is not necessary to assume DP-internal plurality formation to explain examples like this. One alternative way to analyze (39) is to assume that the relevant reading is derived via conjunction reduction from the following sentence (cf.Ross 1967; Hankamer 1979; Hirsch 2017).

Andy wrote a paper and Andy and Patrick wrote a paper.

Another possibility is that the subject DP of (39) is indeed co-extensional with Andy and Patrick, but has an effect on the interpretation of the covert distributivity operator. Schwarzschild (1996), among others, claims that the distributivity operator can distribute a plurality not only into atoms that comprise it, but also into a contextually salient cover of the plurality (A set $\mathscr{C}$ of pluralities is a cover of the plurality $X$ iff $\bigcup \mathscr{C}=X$ ), and crucially a cover can contain overlapping pluralities, e.g. $\{\{a\},\{a, p\}\}$ is a covert of $\{a, p\}$. As Schwarzschild argues, how the plurality is expressed matters for which covers are contextually available. In (39), then, can could assume that the cover $\{\{a\},\{a, p\}\}$ is made available by the form of the subject DP, although the denotation of the subject DP is the same as that of Andy and Patrick, which wouldn't make this cover available. Then we can derive the relevant reading of (39) with the cover-based distributivity operator (which takes scope over the plurality-forming type-shift). We therefore believe that examples like (39) does not necessarily require the mechanism of plurality-formation to be available within DPs.

Coming back to (4b) and (15a), we now understand why they are infelicitous. The predicates here are distributive and therefore, the exclusivity inference of just takes sentential scope, which makes the focus alternatives mutually incompatible, and the additive 
inference of even contradictory. By the same logic, we can account for the lack of minimal sufficiency readings in (1) and (3a). That is, these examples are incompatible with covert even, so cannot have scalar inferences.

\section{Focus alternatives for just}

At this point, we would like to come back to the example in (18) once again. As we mentioned above, this examples has an DP-level exclusivity reading, and as we predict, it is compatible with even, as in (37), reproduced here.

$$
\text { Even just this teapot }{ }_{F} \text { costs } £ 3 \text {. }
$$

What is notable about this example, however, is that it implies that $£ 3$ is expensive, and it turns out that our proposal so far does not account for this. To see this, consider the variant of this example without just.

$$
\text { Even this teapot }{ }_{F} \text { costs } £ 3 \text {. }
$$

This sentence can be felicitously used in contexts where $£ 3$ is considered expensive, but it can also be felicitously used in contexts where it is considered cheap. For instance, suppose that you go into a shop with a friend and see that many items are sold for $£ 3$ there, which both of you find quite cheap. Then you can pick up a teapot and tell your friend (41). By contrast, (37) is utterly infelicitous in this context. Our analysis of just so far cannot explain this contrast, because according to it, the exclusivity inference of just is simply nullified in (37) by the plurality-forming type-shift, so (37) should be able to mean the same thing as (41).

In order to understand the more restricted range of meaning allowed for (37), we propose that just imposes a condition on the set of focus alternatives. Specifically, it triggers a (presuppositional) requirement that the prejacent be a unique bottom element with respect to generalized entailment $\Rightarrow$ in the contextually determined set of alternatives. The idea is that for (37), the alternatives for just have to look like (42), for example.
a. this teapot
b. this teapot and the milk jar
c. this teapot and a teacup
d. this teapot and two teacups
e. this teapot and the milk jar and a teacup
f. this teapot and the milk jar and two teacups

Consider the lifted denotations of these expressions. Then, this teapot is a bottom element with respect to $\Rightarrow$ in the sense that it is entailed by every other focus alternative, and also it is a unique one. By contrast, a set like the following (or any superset of it) is not a legitimate set of focus alternatives for just, under the present analysis.
a. this teapot
b. the milk jar
c. a teacup

Note that the exclusivity inference that just triggers with respect to these two sets of focus alternatives will be the same, namely, in both cases, the denotation will be the generalized quantifier $\lambda P . P(t)=1$ and $P(x)=0$, for any other contextually relevant alternative $x$. Importantly, however, it will have an effect for the inference of even. If the focus alternatives for just are as in (42), those for even will be (44). 
a. just this teapot costs $£ 3$

b. just this teapot and the milk jar cost $£ 3$

c. just this teapot and a teacup cost $£ 3$

d. just this teapot and two teacups cost $£ 3$

e. just this teapot and the milk jar and a teacup cost $£ 3$

f. just this teapot and the milk jar and two teacups cost $£ 3$

These focus alternative are about the prices of different combinations of objects, but crucially, they all involve the same teapot. Let us assume that cost $£ 3$ is understood with the lower-bounded ('at least') semantics (otherwise we would derive an unwanted additive presupposition). In a normal state of affairs buying more things will never decrease the price. Given this, the scalar presupposition of even that (44a) is relatively unlikely/remarkable in these alternatives will be satisfied, if the price is already expensive for (44a). If $£ 3$ were cheap and if (44a) were unlikely/remarkable, on the other hand, then all the other focus alternatives would be at least equally unlikely/remarkable. Therefore the scalar presupposition will not be satisfied.

The version of the sentence without just, (41), on the other hand, has no restrictions on the shape of the set of focus alternatives, and is compatible with the focus alternatives in (42) as well as the ones in (43). In the latter case the focus alternatives for even will look like (45).
a. just this teapot costs $£ 3$
b. just the milk jar costs $£ 3$
c. just a teacup costs $£ 3$

This correctly captures the fact that (41) has wider range of meanings.

It should be made clear, furthermore, that this new assumption will not change our analysis of the minimal sufficiency reading. For examples like (2), the focus alternatives for just are as in (46), and already has a unique bottom element, on the assumption that they receive lower-bounded ('at least') readings (as we assumed in our earlier analysis).
a. one cat
b. two cats
c. three cats

For the other example, (15a), we now have to use conjunctive focus alternatives likes the ones in (47).
a. a letter from her
b. a letter and a phone call from her
c. a letter and a video call from her
d. a letter, a phone call, and a video call from her

The exclusivity inference of just will not change, and moreover, the scalar presupposition of even with respect to the following focus alternatives is a reasonable characterization of the scalar component of the minimal sufficiency reading of (15a).

(48) a. just a letter from her makes me happy

b. just a letter and a phone call from her make me happy

c. just a letter and a video call from her make me happy

d. just a letter, a phone call, and a video call from her make me happy 
One crucial theoretical consequence of this is that the focus alternatives of just can be structurally more complex than the prejacent, contrary to what has been assumed in the literature (see Fox \& Katzir 2011). We believe, however, if it is restricted to conjunctive focus alternatives, it does not create empirical issues, and furthermore it might yield a new insight into different uses of exclusive particles and other focus sensitive particles, although this certainly requires further investigation (see Panizza \& Chierchia 2019 for an analysis of the rank-order reading of exclusive particles in terms of disjunctive focus alternatives).

\section{Conclusion and further issues}

To summarize, the main empirical contribution of the paper is that the distribution of the minimal sufficiency reading of just is correlated with the compatibility with an overt even. This generalization seems to us to be empirically solid and free from exceptions. ${ }^{14}$ We proposed an account that directly captures this generalization by assuming that the minimal sufficiency reading requires a covert even at the sentential level. We furthermore demonstrated that the standard, independently motivated semantics of just and even can be used to understand the distribution of the minimal sufficiency reading, and pointed out the role of (non-)distributivity in this phenomenon. In particular, distributive predicates do not allow for the DP-level exclusivity inference of just, and sentences containing distributive predicates are incompatible with even, and correspondingly, unable to receive minimal sufficiency readings. Our explanation for this is because the sentence-level exclusivity inference of just renders the focus alternatives mutually incompatible, and the additive presupposition of even becomes contradictory.

Before closing, we would like to briefly mention some further issues. Firstly, we assumed all along that just receives an exclusivity reading, but examples like (5) show, it is an empirical fact that it can receive a scalar reading at least in some examples. How and when a scalar reading is observed is an interesting issue, especially in light of our proposal about the constraint on focus alternatives for just proposed in the previous section, but we have to leave this question for future research (see Coppock \& Beaver 2014; Panizza \& Chierchia 2019; Alxatib 2020, for discussion).

Another open issue is that minimal sufficiency readings are quite often observed with just, but they do not seem to be easily available with only, if not impossible. Coppock \& Beaver (2014: 374) raise the following data involving just, only, and other exclusive particles, including cases where an exclusive particle occurs inside the DP, as in (49b).

(49) a. Just/merely/simply the thought of food makes me hungry.

b. The mere thought of food makes me hungry.

c. ?Only the thought of food makes me hungry.

d. \#Exclusively/purely/solely the thought of food makes me hungry.

A similar contrast obtains with numerals. For instance, the following example seems to prefer an exclusivity reading.

Only one cat will make Patrick happy.

\footnotetext{
${ }^{14}$ Grosz (2012) considers examples that involve even and an exclusivity particle, and conclude that minimal sufficiency readings cannot be accounted for in terms of even, but his reasoning hinges on the assumption that optative uses of the exclusive particles are instances of minimal sufficiency readings. We believe his conclusion should be reevaluated in light of our criticism against his analysis of minimal sufficiency readings as well as of our empirical generalization that seems to us to be very robust, but we would like to leave it for another occasion.
} 
Coppock \& Beaver (2014: 401) furthermore gives the following example, where only does give rise to a minimal sufficiency reading:

(51) Only a small percentage would be enough for a big scale scam to take off.

At this moment, we do not have a concrete idea about how to account for this variation among exclusive particles, but we would like to note that (51), but not (50), is compatible with an overt even, so our generalization is still valid here. Similarly, our generalization about overt even holds for the examples in (49). This might suggest that some exclusive particles are subject to further scopal constraints.

\section{Acknowledgements}

We wish to thank the three anonymous reviewers for this journal for constructive comments and criticisms. Our special thanks go to Gennaro Chierchia for helpful comments and suggestions, and to Flóra Lili Donáti for feedback on an earlier version fo this paper. We are also indebted to feedback from the audiences of RALFe 2018 at Université Paris 8 and GLOW 2019 at the University of Oslo, where earlier versions of this work were presented, and the participants of our classes at two summer schools, EGG 2018 at the University of Banja Luka and BLING 2019 at Lake Balaton, where we discussed related ideas. All remaining errors are our own.

\section{Competing Interests}

The authors have no competing interests to declare.

\section{References}

Alxatib, Sam. 2020. Focus, Evaluativity, and Antonymy. Berlin: Springer. DOI: https://doi. org/10.1007/978-3-030-37806-6

Beaver, David \& Brady Clark. 2008. Sense and Sensitivity: How Focus Determines Meaning. Wiley-Blackwell. DOI: https://doi.org/10.1002/9781444304176

Breheny, Richard. 2008. A new look at the semantics and pragmatics of numerically quantified noun phrases. Journal of Semantics 25(2). 93-139. DOI: https://doi. org $/ 10.1093 /$ jos/ffm016

Champollion, Lucas. 2016. Ten men and women got married today: Noun coordination and the intersective theory of conjunction. Journal of Semantics 33(3). 561-622. DOI: https://doi.org/10.1093/jos/ffv008

Champollion, Lucas. 2017. Parts of a Whole: Distributivity as a Bridge Between Aspect and Measurement. Oxford: Oxford University Press. DOI: https://doi.org/10.1093/ oso/9780198755128.003.0009

Chierchia, Gennaro. 2013. Logic in Grammar: Polarity, Free Choice, and Intervention. Oxford: Oxford University Press. DOI: https://doi.org/10.1093/acprof: oso/9780199697977.001.0001

Chierchia, Gennaro, Danny Fox \& Benjamin Spector. 2012. Scalar implicature as a grammatical phenomenon. In Claudia Maienborn, Klaus von Heusinger \& Paul Portner (eds.), Semantics: An International Handbook of Natural Language Meaning, 2297-2331. Berlin: de Gruyter.

Coppock, Elizabeth \& David Beaver. 2014. Pirnciples of the Exclusive Muddle. Journal of Semantics 31(3). 371-432. DOI: https://doi.org/10.1093/jos/fft007

Crnič, Luka. 2011. Getting Even. Massachusetts Institute of Technology Ph.D. dissertation. 
Fox, Danny. 2007. Free choice and the theory of scalar implicatures. In Uli Sauerland \& Penka Stateva (eds.), Presupposition and Implicature in Compositional Semantics, 71-112. Palgrave Macmillan. DOI: https://doi.org/10.1057/9780230210752_4

Fox, Danny \& Roni Katzir. 2011. On the characterization of alternatives. Natural Language Semantics 19(1). 87-107. DOI: https://doi.org/10.1007/s11050-010-9065-3

Geurts, Bart. 2006. Take 'five': The meaning and use of a number word. In Nondefiniteness and plurality. John Benjamins. DOI: https://doi.org/10.1075/la.95.16geu

Geurts, Bart. 2010. Quantitiy Implicatures. Cambridge: Cambridge University Press. DOI: https://doi.org/10.1017/CBO9780511975158

Greenberg, Yael. 2018. A revised, gradability-based semantics for even. Natural Language Semantics 26(1). 51-83. DOI: https://doi.org/10.1007/s11050-017-9140-0

Groenendijk, Jeroen \& Martin Stokhof. 1984. Studies on the Semantics of Questions and the Pragmatics of Answers. University of Amsterdam dissertation.

Grosz, Patrick. 2012. On the Grammar of Optative Constructions. Amsterdam: John Benjamins. DOI: https://doi.org/10.1075/la.193

Guerzoni, Elena. 2003. Why Even Ask? On the Pragmatics of Questions and the Semantics of Answers. Massachusetts Institute of Technology Ph.D. dissertation.

Hankamer, Jorge. 1979. Deletion in Coordinate Structures. New York: Garland.

Herburger, Elena. 2000. What Counts: Focus and Quantification. Cambridge, MA: MIT Press. DOI: https://doi.org/10.7551/mitpress/7201.001.0001

Hirsch, Aron. 2017. An Inflexible Semantics for Cross-Categorial Operators. Massachusetts Institute of Technology dissertation.

Horn, Larry. 1969. A presuppositional analysis of only and even. In CLS 4. 98-108.

Horn, Laurence. 1972. On the Semantic Properties of the Logical Operators. University of California Los Angeles dissertation.

Ippolito, Michela. 2008. On the meaning of only. Journal of Semantics 25. 45-91. DOI: https://doi.org/10.1093/jos/ffm010

Karttunen, Lauri \& Stanley Peters. 1979. Conventional implicature. In Choon-Kyu Oh \& David Dinneen (eds.), Syntax and Semantics 11: Presupposition, 1-56. New York: Academic Press.

Kay, Paul. 1990. Even. Linguistics and Philosophy 13(1). 59-111. DOI: https://doi. org/10.1007/BF00630517

Krifka, Manfred. 1991. A compositonal semantics for multiple focus constructions. In Proceedings of SALT 1. DOI: https://doi.org/10.3765/salt.v1i0.2492

Lahiri, Utpal. 1998. Focus and negative polarity in Hindi. Natural Language Semantics 6. 57-125. DOI: https://doi.org/10.1023/A:1008211808250

Mayr, Clemens \& Jacopo Romoli. 2016. A puzzle for theories of redundancy: Exhaustification, incrementality, and the notion of local context. Semantics and Pragmatics 9(7). 1-48. DOI: https://doi.org/10.3765/sp

Meyer, Marie-Christine. 2016. Redundancy and embedded exhaustification. In Proceedings of SALT 25. DOI: https://doi.org/10.3765/salt.v25i0.3486

Nakanishi, Kimiko. 2006. Even, only, and negative polarity in Japanese. In SALT 16. DOI: https://doi.org/10.3765/salt.v16i0.2953

Panizza, Daniele \& Gennaro Chierchia. 2019. Just exhaustification: A 'two stage' theory of exclusives. In Proceedings of the 22nd Amsterdam Colloquium 2019, 337-346.

Roberts, Craige. 1987. Modal Subordination, Anaphora and Distributivity. University of Massachusetts, Amherst Ph.D. dissertation.

Rooth, Mats. 1985. Association with Focus. University of Massachusetts, Amherst Ph.D. dissertation. 
Rooth, Mats. 1992. A theory of focus interpretation. Natural Language Semantics 1(1). 75-116. DOI: https://doi.org/10.1007/BF02342617

Ross, John. 1967. Constraints on Variables in Syntax. Massachusetts Institute of Technology dissertation.

Rullmann, Hotze. 1997. Even, polarity, and scope. In Papers in Experimental and Theoretical Linguistics, vol. 4, 40-64. University of Alberta Working Papers in Linguistics.

Rullmann, Hotze. 2007. What does even even mean? Ms., University of British Columbia.

Russell, Benjamin. 2006. Against grammatical computation of scalar implicatures. Journal of Semantics 23(4). 361-382. DOI: https://doi.org/10.1093/jos/ffl008

Sauerland, Uli. 2004. Scalar implicatures in complex sentences. Linguistics and Philosophy 27(3). 367-391. DOI: https://doi.org/10.1023/B:LING.0000023378.71748.db

Schwarzschild, Roger. 1996. Pluralities. Dordrecht: Kluwer. DOI: https://doi. org/10.1007/978-94-017-2704-4

Spector, Benjamin. 2013. Bare numerals and scalar implicatures. Language and Linguistics Compass 7(5). 273-294. DOI: https://doi.org/10.1111/lnc3.12018

Sudo, Yasutada. 2017. It's not always redundant to assert what can be presupposed. Ms., University College London.

Wilkinson, Karina. 1996. The scope of even. Natural Language Semantics 4(3). 193-215. DOI: https://doi.org/10.1007/BF00372819

Winter, Yoad. 2001. Flexibility Principles in Boolean Semantics: The Interpretation of Coordination, Plurality, and Scope in Natural Language. Cambridge, MA: MIT Press. DOI: https://doi.org/10.7551/mitpress/3034.001.0001

How to cite this article: Panizza, Daniele and Yasutada Sudo. 2020. Minimal sufficiency with covert even. Glossa: a journal of general linguistics 5(1): 98.1-25. DOI: https://doi.org/10.5334/gjgl.1118

Submitted: 30 September 2019

Accepted: 05 July 2020

Published: 06 October 2020

Copyright: $\odot 2020$ The Author(s). This is an open-access article distributed under the terms of the Creative Commons Attribution 4.0 International License (CC-BY 4.0), which permits unrestricted use, distribution, and reproduction in any medium, provided the original author and source are credited. See http://creativecommons.org/licenses/by/4.0/.

$\mathrm{u}[\quad$ Glossa: a journal of general linguistics is a peer-reviewed open access journal published by Ubiquity Press.

OPEN ACCESS $\precsim$ 